\title{
The Effects of Voiding Habits on Uroflowmetry in Childhood
}

\author{
Mehran Hiradfar, ${ }^{1}$ Ehsan Habibpur, ${ }^{2}$ Parisa Saeedi Sharifabad, ${ }^{3}$ Reza Shojaeian, ${ }^{4,}$ Habibollah \\ Esmaeeli, ${ }^{5}$ and Fatemeh Ghane ${ }^{6}$ \\ ${ }^{1}$ Associate Professor of Pediatric Surgery, Endoscopic and Minimally Invasive Surgery Research Center, Ghaem Hospital, Faculty of Medicine, Mashhad University of Medical \\ Sciences, Mashhad, Iran \\ ${ }^{2}$ Medical Student, Researcher, Mashhad University of Medical Sciences, Mashhad, Iran \\ ${ }^{3}$ Assistant Professor of Pediatric Urology, Department of Pediatric Urology, Mashhad University of Medical Sciences, Mashhad, Iran \\ ${ }^{4}$ Assistant Professor of Pediatric Surgery, Endoscopic and Minimally Invasive Surgery Research Center, Ghaem Hospital, Faculty of Medicine, Mashhad University of Medical \\ Sciences, Mashhad, Iran \\ ${ }^{5}$ Professor of Pediatric Nephrology, Department of Pediatric Nephrology, Mashhad University of Medical Sciences, Mashhad, Iran \\ ${ }^{6}$ Associate Professor of Pediatric Nephrology, Department of Pediatric Nephrology, Mashhad University of Medical Sciences, Mashhad, Iran \\ "Corresponding author: Reza Shojaeian, Assistant Professor of Pediatric Surgery, Endoscopic and Minimally Invasive Surgery Research Center, Ghaem Hospital, Faculty of \\ Medicine, Mashhad University of Medical Sciences, Mashhad, Iran. Tel: +98-9155150923, E-mail: drshojaeian@ymail.com
}

Received 2016 September 23; Revised 2016 November 20; Accepted 2016 December 27.

\begin{abstract}
Background: Urodynamic studies (UDS) is a simple non-invasive test to assess lower urinary tract function but it may be affected by several factors includes age, sex, voiding volume and voiding position. These interventional parameters become more highlighted while performing UDS in children whose emotional stress control and coping with unfamiliar situation is more difficult.

Methods: Seventy six primary school age healthy children underwent screening uroflowmetry in different voiding positions voluntarily. Cases with urinary symptoms, other coexisting diseases, voiding volume less than 20cc or staccato voiding curve were excluded. Washrooms were designed in both western and eastern styles. UDS indexes were compared regarding voiding habits.

Results: Comparison of uroflowmetric indexes between different genders and voiding positions showed differences which were not statistically significant but considering the voiding habits, uroflowmetric indexes were significantly different in familiar compare to unfamiliar voiding position. Q-Max was lower and time to Q-Max and micturition time were longer in unfamiliar voiding position. Conclusions: Urodynamic studies in children should be performed in preferred voiding position for each kid considering the culture and voiding habits. Unfamiliar voiding position may turn the uroflowmetry to a stressful experience for child that make him uncooperative and cause misleading results.
\end{abstract}

Keywords: Urology, Pediatric, Urodynamic, Voiding Dysfunction

\section{Background}

Urodynamic studies (UDS) has become one of the most popular non-invasive diagnostic tools in the evaluation of lower urinary tract function. It provides valuable information about urine storage and micturition in an easy costeffective way (1). Uroflowmetry assesses urination function through measurement of total urine volume, mean and maximum urine flow rates. Reliable measurements however, depend on patient cooperation, investigator skills, and equipment calibration (2).

It is established that uroflowmetric indexes are affected by several factors such as age, sex, voiding volume, subject's knowledge of the experiment's procedure, and voiding position (3). These interventional parameters are more significant in pediatric population, as their emotional stress control and their ability to cope with unfamiliar situations is underdeveloped.

The role of voiding position in uroflowmetry is discussed in literature. Higher flow rates are observed in standing and squatting positions $(4,5)$, although some controversies still exist regarding the pediatric population $(6,7)$. There are some limitations in the evaluation of voiding positions in uroflowmetry. One such limitation is the effect of stressful voiding in an unfamiliar situation. This may cause hesitancy and affect uroflowmetry significantly (3). Another limitation is the fact that urodynamic equipment is assembled according to the western culture and standards, whereas voiding styles can differ considerably according to culture and social norms. This article assesses the effect of voiding habits on the results of uroflowmetry in the pediatric population.

\section{Methods}

Randomized sampling was performed among 7-9 year old healthy children in primary schools in Mashhad, Iran, in 2006. The medical ethics committee of Mashhad University of Medical Sciences approved this study as a noninvasive screening test for healthy volunteers. 
Information regarding what to expect during and after the screening test was explained in simple terms and illustrations for parents. Consent forms were signed by participants' parents or legal guardians.

Our exclusion criteria included refuse to participate or lack of patient cooperation, previous operation on urinary tract, known urinary tract disease like hypospadiasis or meatal stenosis, history of urinary symptoms such as incontinence, dribbling and dysuria, or any related neurological problem such as cerebral palsy or myelomeningocele. Those patients who failed to complete the study or those with total urine volume less than $20 \mathrm{cc}$ or staccato patern or interrupted voiding curve were also excluded. Informed

In this study, Dantec Duet $₫$ 8.06 (Dantec Medical, Denmark) uroflowmeter was used. Participants were asked to come in two times a day with a full bladder, and were assigned to bathrooms that contained both eastern and western style toilets. The western style (sitting position) was the standard uroflowmetric equipment, whereas the eastern style (squatting position) was simulated for our trial in urodynamic department. The assignment of eastern or western test was determined randomly for each participant. A trained urodynamic nurse was blinded to each subject's voiding style.

There were 76 participants who completed the study. Participants whose total urine volume were less than $20 \mathrm{cc}$ or those with staccato or interrupted voiding curve were excluded. The data that was collected included: void volume, maximum flow rate ( $\mathrm{Q}$ Max), mean flow rate ( $\mathrm{Q}$ average) time to maximum flow rate, and voiding time.

Post-voiding residual urine was evaluated by transabdominal ultrasound at the end of each uroflowmetry session. Data was stratified according to gender, micturition habits, and voiding position. Analysis of variance (ANOVA) and Tukey test by SPSS 11.5 were utilized to compare data between groups.

\section{Results}

76 cases out of a total of 300 participants were completed the trail and fit the inclusion criteria properly. Among these children, 49 (64.5\%) were boys with the mean age of $8.3 \pm 1.7$ and 27 (35.5\%) were girls with the mean age of $9.1 \pm 2.4$ years. At home, out of a total of 76 participants, only 13 children (17\%) [ 8 boys and 5 girls] used a western style of voiding (sitting position) while the other 63 participants [ 41 boys and 22 girls] used eastern voiding style (squatting position).

Comparing uroflowmetric indexes in eastern and western voiding positions among both genders (Table 1), we observed higher voided volume, mean and max flow rate in squatting position (eastern style) while shorter time to Q max and longer voiding time in eastern position. However, the differences were not statistically significant.

Considering the voiding habits, uroflowmetric indexes were compared in familiar and unfamiliar voiding positions among boys and girls. Higher voided volume, mean and max flow rate and longer voiding time were observed in familiar position. Time to Q Max was significantly longer among boys in and lag time was also significantly longer in unfamiliar position among both girls and boys (Table 2).

Post-voiding residual volume was evaluated by ultrasound in all cases and mean residual volume was $16.7 \pm 9.5$ $\mathrm{mL}$ in eastern position, $23.7 \pm 15.6 \mathrm{~mL}$ in western position, $16.1 \pm 8.9 \mathrm{~mL}$ in familiar voiding position, and $25.1 \pm 17.3$ $\mathrm{mL}$ in unfamiliar voiding position. Therefore, the highest residual volume was observed in unfamiliar voiding position.

\section{Discussion}

Urodynamic studies (UDS) is a valuable non-invasive tool for functional assessment of bladder and urethra. Nonetheless, incorrect equipment set up and test performance may result in false data.

Several studies have assessed the role of voiding position on uroflowmetry in adults and the results are controversial. While some studies do not see any correlation between voiding position and flow rates $(6,7)$, other studies have demonstrated how voiding positions can significantly influence urodynamic indexes $(1,5,8,9)$. However, there is still no consensus on the role of any voiding position on a specific UDS index.

In this study we selected a group of healthy volunteer children to participate in a screening urodynamic study involving different voiding positions. The most common voiding position in eastern cultures is the squatting position, while standard UDS devices are assembled for use in sitting voiding position.

We considered the interrupted voiding pattern or staccato curve as exclusion criteria. All the volunteers were asymptomatic healthy children with no history of urinary tract problems. Post voiding ultrasound studies were also normal in all the cases. We had five cases with abnormal or staccato pattern (among total 81 volunteers) that count as $6.2 \%$, which is a high rate among healthy normal population. After reviewing the questionnaires regarding voiding positions, it was realized that all the abnormal patterns were recorded in UDS during unfamiliar voiding positions.

Our findings highlighted the importance of familiar voiding position while performing UDS especially in children. Uncomfortable voiding position and stress may even 
Hiradfar M et al.

Table 1. Comparing Uroflowmetric Indexes in Western and Eastern Voiding Positions Among Different Genders

\begin{tabular}{|c|c|c|c|c|}
\hline Uroflowmetric Indexes & Gender & Western Voiding Position & Eastern Voiding Position & $\mathbf{P}^{\mathbf{a}}$ \\
\hline \multirow{2}{*}{ Voided volume (mL) } & Boys & $131.4 \pm 58.9$ & $146.6 \pm 82.5$ & 0.69 \\
\hline & Girls & $123.6 \pm 81.4$ & $129.7 \pm 92.4$ & 0.78 \\
\hline \multirow{2}{*}{ Mean flow rate $(\mathrm{mL} / \mathrm{s})$} & Boys & $9.4 \pm 3.0$ & $9.7 \pm 5.1$ & 0.86 \\
\hline & Girls & $10.1 \pm 3.4$ & $11.3 \pm 3.6$ & 0.73 \\
\hline \multirow{2}{*}{ Max flow rate (mL/s) } & Boys & $18.3 \pm 6.1$ & $20.5 \pm 6.8$ & 0.51 \\
\hline & Girls & $22.6 \pm 7.4$ & $25.2 \pm 12.2$ & 0.64 \\
\hline \multirow{2}{*}{ Time to Q max (sec) } & Boys & $5.6 \pm 3.4$ & $4.4 \pm 2.8$ & 0.37 \\
\hline & Girls & $4.7 \pm 4.1$ & $4.1 \pm 1.9$ & 0.41 \\
\hline \multirow{2}{*}{ voiding time (s) } & Boys & $14.2 \pm 9.1$ & $17.6 \pm 7.2$ & 0.11 \\
\hline & Girls & $11.4 \pm 9.8$ & $15.8 \pm 9.5$ & 0.28 \\
\hline \multirow{2}{*}{ Lag time (sec) } & Boys & $3.1 \pm 1.4$ & $2.4 \pm 1.8$ & 0.45 \\
\hline & Girls & $2.7 \pm 1.9$ & $1.9 \pm 0.9$ & 0.51 \\
\hline
\end{tabular}

${ }^{\mathrm{a}} \mathrm{T}$ test $\mathrm{P}$ value.

Table 2. Comparing Uroflowmetric Indexes Between Familiar and Unfamiliar Voiding Positions Among Different Genders

\begin{tabular}{|c|c|c|c|c|}
\hline Uroflowmetric Indexes & Gender & Unfamiliar Voiding Position & Familiar Voiding Position & $\mathbf{P}^{\mathbf{a}}$ \\
\hline \multirow{2}{*}{ Voided volume (mL) } & Boys & $129.1 \pm 49.9$ & $148.9 \pm 97.6$ & 0.19 \\
\hline & Girls & $120.1 \pm 95.4$ & $133.2 \pm 106.1$ & 0.24 \\
\hline \multirow{2}{*}{ Mean flow rate $(\mathbf{m L} / \mathbf{s})$} & Boys & $9.1 \pm 3.2$ & $10.1 \pm 4.8$ & 0.37 \\
\hline & Girls & $9.9 \pm 4.4$ & $11.5 \pm 4.1$ & 0.66 \\
\hline \multirow{2}{*}{ Max flow rate $(\mathrm{mL} / \mathbf{s})$} & Boys & $18.1 \pm 6.5$ & $20.7 \pm 6.4$ & 0.11 \\
\hline & Girls & $22.2 \pm 6.9$ & $25.6 \pm 10.1$ & 0.24 \\
\hline \multirow{2}{*}{ Time to Q max (sec) } & Boys & $5.9 \pm 4.2$ & $4.1 \pm 3.2$ & $<0.05$ \\
\hline & Girls & $4.9 \pm 3.7$ & $4.0 \pm 2.1$ & 0.08 \\
\hline \multirow{2}{*}{ voiding time (s) } & Boys & $15.6 \pm 8.5$ & $16.2 \pm 9.3$ & 0.31 \\
\hline & Girls & $12.1 \pm 7.6$ & $14.9 \pm 10.1$ & 0.61 \\
\hline \multirow{2}{*}{ Lag time (sec) } & Boys & $3.7 \pm 1.8$ & $1.8 \pm 2.01$ & $<0.05$ \\
\hline & Girls & $3.2 \pm 1.6$ & $1.5 \pm 0.8$ & $<0.05$ \\
\hline
\end{tabular}

${ }^{\mathrm{a}} \mathrm{T}$ test $\mathrm{P}$ value.

lead to false results such as staccato pattern even in a healthy child.

Comparison of urodynamic indexes in different voiding positions revealed significant changes in lag time and time to max flow among children in unfamiliar voiding position, so we concluded that although sitting or squatting voiding positions may affect the UDS results, unfamiliar voiding position is the main factor that may alter the results of UDS in children. Importance of familiar voiding position in UDS was also reported in young adults previously $(4,8,9)$ and articles have recommended performing uroflowmetry in preferred position of individuals (9).

Our study showed more significant role of voiding habits in pediatrics may be due to immature coping and stress control ability in pediatrics. Unfamiliar voiding position or lack of privacy may make the UDS a stressful experience for a child. This may affect the indexes, especially hesitancy (lag time) and time to Q max significantly. It may also cause incomplete bladder emptying or interrupted or staccato curve.

The issue will become more important considering the growing rate of immigration, health tourism and growing 
of new multi nationality communities.

Conclusion: Urodynamic studies in children should be performed in their preferred voiding position. Unfamiliar voiding position and stress may cause misleading results. Simulation of UDS devices considering the culture and voiding habits may help obtain more accurate results.

\section{Acknowledgments}

Special thanks to Ms Vafa, Urodynamic department technician for her kind coaporation in conducting the uroflometeries and Mrs Maryam Hiradfar for her helps in English editing of the manuscript.

\section{Footnotes}

Financial Disclosure: Nothing to disclose.

Funding/Support: This study carried out with research grant of the first author and supports of minimally invasive and endoscopic surgery research center and research council of Mashhad University of medical sciences.

\section{References}

1. Koc G, Yilmaz Y, Un S, Akbay K, Akdeniz F. The effect of alpha blockers on uroflowmetric parameters in different voiding positions. Can Urol Assoc J. 2013;7(5-6):E329-32. doi:10.5489/cuaj.12091. [PubMed: 23766834].
2. Kajbafzadeh AM, Yazdi CA, Rouhi O, Tajik P, Mohseni P. Uroflowmetry nomogram in Iranian children aged 7 to 14 years. BMC Urol. 2005;5:3. doi: 10.1186/1471-2490-5-3. [PubMed: 15771771].

3. Uluocak N, Oktar T, Acar O, Incesu O, Ziylan O, Erkorkmaz U. Positional changes in voiding dynamics of children with nonneurogenic bladder dysfunction. Urology. 2008;72(3):530-4. doi: 10.1016/j.urology.2008.02.067. [PubMed: 18502486] discussion 534-5.

4. Choudhury S, Agarwal MM, Mandal AK, Mavuduru R, Mete UK, Kumar $\mathrm{S}$, et al. Which voiding position is associated with lowest flow rates in healthy adult men? role of natural voiding position. Neurourol Urodyn. 2010;29(3):413-7. doi: 10.1002/nau.20759. [PubMed: 19634168].

5. Aghamir SM, Mohseni M, Arasteh S. The effect of voiding position on uroflowmetry findings of healthy men and patients with benign prostatic hyperplasia. Urol J. 2005;2(4):216-21. [PubMed: 17602433].

6. Unsal A, Cimentepe E. Voiding position does not affect uroflowmetric parameters and post-void residual urine volume in healthy volunteers. Scand J Urol Nephrol. 2004;38(6):469-71. [PubMed: 15841779].

7. Unsal A, Cimentepe E. Effect of voiding position on uroflowmetric parameters and post-void residual urine volume in patients with benign prostatic hyperplasia. Scand J Urol Nephrol. 2004;38(3):240-2. doi: 10.1080/00365590410028674. [PubMed: 15204379].

8. Eryildirim B, Tarhan F, Kuyumcuoglu U, Erbay E, Pembegul N. Positionrelated changes in uroflowmetric parameters in healthy young men. Neurourol Urodyn. 2006;25(3):249-51. doi: 10.1002/nau.20221. [PubMed: 16496389].

9. El-Bahnasawy MS, Fadl FA. Uroflowmetric differences between standing and sitting positions for men used to void in the sitting position. Urology. 2008;71(3):465-8. doi: 10.1016/j.urology.2007.11.011. [PubMed: 18342188]. 